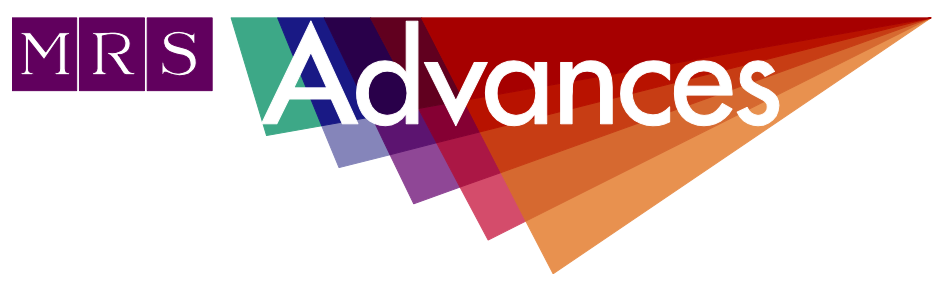

\title{
Activity of photosynthetic Reaction Centers coated with polydopamine
}

\author{
Francesco Milano ${ }^{1}$, Marco Lopresti ${ }^{2}$, Danilo Vona, ${ }^{2}$ Gabrielle Buscemi ${ }^{2,3}$, Mariangela \\ Cantore $^{3}$, Gianluca M. Farinola ${ }^{2}$, Massimo Trotta ${ }^{3}$
}

${ }^{I}$ Istituto di Scienze delle Produzioni alimentari CNR-ISPA, Via Prov.le Monteroni, 73100 Lecce ${ }^{2}$ Dipartimento di Chimica, Università degli Studi di Bari "Aldo Moro", via Orabona 4, I-70126 Bari; ${ }^{3}$ Istituto per i Processi Chimico-Fisici CNR-IPCF, Dipartimento di Chimica, via Orabona 4, I-70126 Bari;

Dilute aqueous solution of dopamine buffered to an alkaline $\mathrm{pH}$ and in the presence of dissolved oxygen undergo to a series of autoxidation and rearrangement reactions that lead to the formation of a dark insoluble material called polydopamine (PDA) with melanin reminiscent properties. In this work we carried out this reaction in the presence of the photosynthetic reaction center $(R C)$, a transmembrane pigment-protein complex responsible for the first light-induced reactions in the photosynthetic process. We have found that PDA grows in colloidal form around the RC and in the appropriate conditions the protein is entrapped in the PDA matrix without loss of functionality. The protein is still capable to perform its natural photocycle leading to the generation of photocurrents and the ubiquinone acceptor complex function is modulated by the PDA/RC ratio.

\section{INTRODUCTION}

In recent years, the field of nanobiocatalysis with immobilized enzymes[1] or living microorganisms[2-3] has gained great attention thanks to the improved techniques to increase stability and activity of the biologacl catalysts. Several nanocarriers have been proposed so far, with porous structure and large surface/area ratio such as $\mathrm{SiO}_{2}$ microparticles with carbon nanotube-derived mesopores[4], magnetic organosilica nanoflowers[5], protein-containing metal-organic frameworks-based hollow composites.[6] The enzyme activity is in many cases enhanced thanks to high loading and large particle surface/volume ratio.

In this framework, it is interesting to exploit the unique characteristic of polydopamine, a melanin-reminiscent dark and insoluble material formed by oxidative polymerization of alkaline solutions of its precursor dopamine[7]. The structure of polydopamine is heavily debated in literature [8-9] but some key points are widely accepted: the self-polymerization starts under weakly basic conditions via successive oxidation, followed by intramolecular 
cyclization, oligomerization and self-assembly, resulting in the highly cross-linked PDA final structure. The final solution results in a slow sedimenting colloidal suspension of PDA nanoaggregates stable for hour or days; moreover, PDA nanoaggregates adhere to the surface of the reaction vessel or to any substrate dipped in solution. The PDA films are largely studied in literature as surface coating material able to entrap active enzymes[10] and amenable to further functionalization exploiting their versatile chemical reactivity of the exposed catechol groups[11]. In this work, rather than to the adhesive PDA films, we focus our attention on the above mentioned PDA colloidal suspensions. Indeed, during the polymeric growth, PDA nanoaggregates can entrap any material present in solution as already demonstrated for the Tris buffer itself [12]. We carried out dopamine polymerization reaction in a slightly alkaline solution containing the photosynthetic reaction center (RC) photoenzyme, a natural photoconverter present in all photosynthetic organisms, with increasing complexity passing from photosynthetic bacteria to algae and plants. The RC used in this work is from the purple photosynthetic bacterium Rhodobacter (R.) sphaeroides strain R26 and is widely accepted in the photosynthetic field as a model system. This enzyme catalyses the endergonic electron transfer reaction from the soluble cytochrome $c_{2}$ (cyt) to the membrane soluble ubiquinone-10 $\left(\mathrm{UQ}_{10}\right)$ exploiting the energy input given by absorbed photons. In isolated proteins, instead, light absorption promotes the formation of an electron-hole couple with a conversion yield close to unity, making the $\mathrm{RC}$ a biological semiconductor[13]. Given the intense research of such kind of proteins in material science for energy conversion[1, 14], synthetic biology[15], biosensing[16-17], and photocatalysis[18], it results interesting to study the behaviour of RCs once entrapped within the suspended PDA nanoaggregates. The RC photoactivity was studied as a function of the starting enzyme/dopamine starting ratio verifying its entrapment without loss of functionality. The ability to produce photocurrents in the presence of diffusive mediators was finally assessed.

\section{EXPERIMENTAL DETAILS}

\section{$\underline{\text { Chemicals }}$}

All chemicals were purchased at the highest purity available and used without further purification. Dopamine hydrochloride (DA), ubiquinone-10 (UQ $\left.\mathrm{U}_{10}\right)$, decylubiquinone (dQ), ferrocenemethanol (FcnMeOH), Triton X-100 (TX), tris(hydroxymethyl) aminomethane (Tris), ethylenediaminetetraacetic acid (EDTA), Fluorescein isothiocyanate (FITC), were purchased from Sigma. Lauryldimethylamine N-oxide (LDAO) was purchased from Fluka. Bidistilled water was used in all experiments. FITClabelled RCs were prepared as previously described[19].

\section{$\underline{\text { Reaction centres }}$}

Reaction Centers were purified from the purple photosynthetic bacterium $R$. sphaeroides strain R26 following the procedure described by Isaacson [20] with minor modifications[21]. Protein purity measured using the absorbance ratio at $280 \mathrm{~nm}\left(\mathrm{~A}_{280}\right)$ and $802 \mathrm{~nm}\left(\mathrm{~A}_{802}\right)$, that was kept at $\mathrm{A}_{280} / \mathrm{A}_{802}<1.4$ while the absorbance ratio $\mathrm{A}_{760} / \mathrm{A}_{865}$ was kept $\leq 1$. The average quinone content was $\mathrm{UQ}_{10} / \mathrm{RC}=1.8$. 
Reaction center spectra were recorded using a UV-vis-NIR Cary 5000 spectrophotometer (Varian Inc. - Australia). Charge recombination kinetics were recorded at $865 \mathrm{~nm}$ using a flash photolysis setup of local design described elsewhere[22]. The sample temperature was set at $25^{\circ} \mathrm{C}$ by a circulating water bath thermo cryostat.

\section{Preparation of RC@PDA suspension}

Entrapment of RCs into the PDA nanoaggregates (RC@PDA) was performed in a mixture of $1.5 \mu \mathrm{M}$ protein, $0.2-20 \mathrm{mM}$ DA in Tris $10 \mathrm{mM}$, LDAO $0.03 \%$, EDTA $1 \mathrm{mM} \mathrm{pH} 8.0$ (TLE buffer). The solution was stirred overnight at room temperature in an open vessel to allow the oxygen and alkaline-mediated DA polymerization. The suspension of entrapped proteins within the growing dark colloidal polymeric aggregates was eventually ultracentrifuged at $33000 \times \mathrm{g}$ for $1 \mathrm{~h}$ at $4{ }^{\circ} \mathrm{C}$. Sedimented nanoaggregates were resuspended in the same buffer for further studies. Supernatants were also analyzed spectrophotometrically to check any residual protein content.

\section{Electrochemical measurements.}

Photoelectrochemical measurements were conducted by an Autolab potentiostat PGSTAT 10 in the three-electrode configuration. The cell was a plastic cuvette $\left(1 \times 1 \mathrm{~cm}^{2}\right.$ base and $1.5 \mathrm{~cm}$ height). The working electrode (WE) was an ITO-covered glass slide; a micro $\mathrm{Ag} / \mathrm{AgCl}$ electrode was used as reference electrode (RE) and a platinum wire used as counter-electrode (CE). The cuvette was filled with $1 \mathrm{~mL}$ of phosphate $100 \mathrm{mM}$, TX-100 $0.03 \% \mathrm{pH} 7.0\left(\mathrm{P}_{100} \mathrm{TX}_{0.03}\right)$ as support electrolyte. The immersed WE surface was $0.9 \mathrm{~cm}^{2}$. FcnMeOH $300 \mu \mathrm{M}$ as electron donor and dQ $100 \mu \mathrm{M}$ as electron acceptor were added in solution together with the RC@PDA suspension. A bias of $-0.1 \mathrm{~V}$, corresponding to the dark OCV of the cell, was applied. The ITO-covered side of the WE was illuminated with a 2.6 W LED (Osram model SFH4783) emitting at $865 \mathrm{~nm}$.

\section{RESULTS AND DISCUSSION}

Reaction Centers (RCs) are specialized pigment-protein transmembrane complexes that promote the conversion of light energy into chemical free energy in the photosynthetic process [23]. The RC isolated from Rhodobacter $(R$.) sphaeroides, contains three protein subunits ( $\mathrm{L}, \mathrm{M}$ and $\mathrm{H}$ ) non-covalently bound to a series of pigments and cofactors involved in the light induced electron transfer reactions[24-25]. RC is vectorially immersed in the membrane separating the periplasmic space delimited by the outer membrane and the inner cytoplasmic space. A bacteriochlorophyll (BChl) dimer that acts as the primary donor (D) is located close to the periplasmic side of the protein. Moving towards the cytoplasmic side, the remaining cofactors are arranged in two symmetric branches A and B. These cofactors are two accessory BChls, two bacteriopheophytins (BPhe) and two ubiquinone$10\left(\mathrm{UQ}_{10}\right)$ molecules forming the so-called quinone acceptor complex $\mathrm{Q}$. The presence of these pigments and cofactors results in several intense absorption peaks in a wide spectral range from 240 to $900 \mathrm{~nm}$. Focusing on the NIR part of the spectrum, the peak centered around $865 \mathrm{~nm}$ originates from $\mathrm{D}$, the peak at $802 \mathrm{~nm}$ from the monomeric BChls, and the peak at $760 \mathrm{~nm}$ from the BPhe. The ratio of the absorption peaks at 760, 802, and $865 \mathrm{~nm}$ is $1: 2: 1$ in the native intact protein, but may slightly change in the solubilizing environment; denaturation processes also heavily modify the ratios.

Hybrid RC@PDA systems were prepared exploiting the natural tendency of the DA to polymerize in basic environments grafting on the surfaces, in particular in the presence of amino groups [26]. In this way, the RC serves as a nucleation site during polymerization 
of DA and remains included inside the nanospheres. Not knowing a priori the stoichiometry of the reaction and the activity or the efficiency of the RC as a function of the amount of used DA, we have initially carried out the polymerization reaction as function of DA hydrochloride concentrations in the presence of a fixed amount of RC.

The spectra of the suspensions recorded after incubation (Figure 1) show the characteristic peaks of the RC superimposed to the scattering signal of the colloidal PDA nanoaggregates. The main peaks of the $\mathrm{RC}$ can be isolated by scattering baseline correction Even at the highest DA concentration used, the typical RC spectrum can be obtained after scattering baseline correction. The1:2:1 peak ratio indicates that no denaturation occurs during PDA formation.

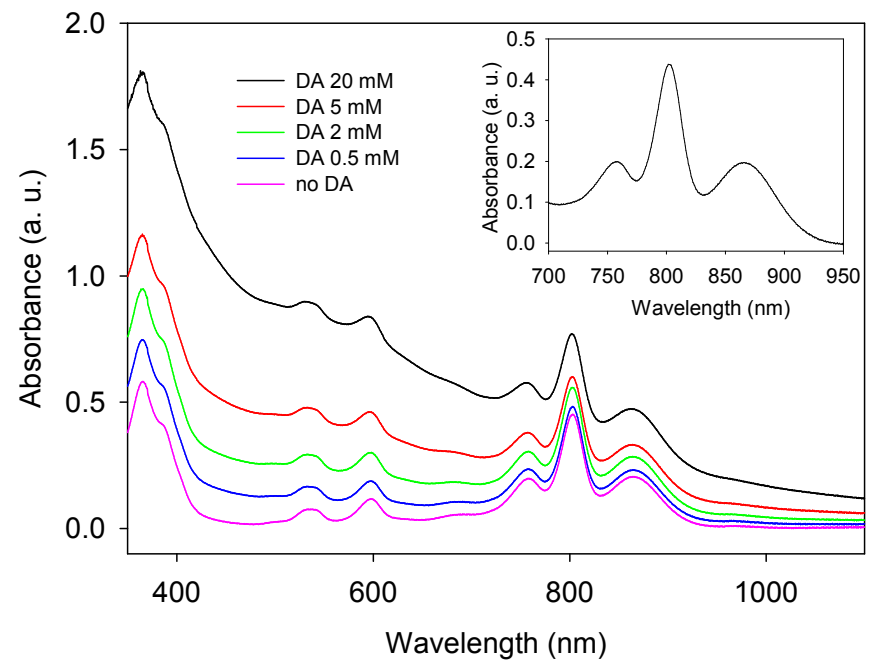

Figure 1. Spectra of RC@PDA suspensions after overnight incubation with different DA concentrations as detailed in the legend. Inset: RC@PDA spectrum with DA 20 mM after baseline correction. See text for details.

The actual RC encapsulation within PDA nanoaggregates was studied through TEM observation without any contrasting agent (Figure 2).
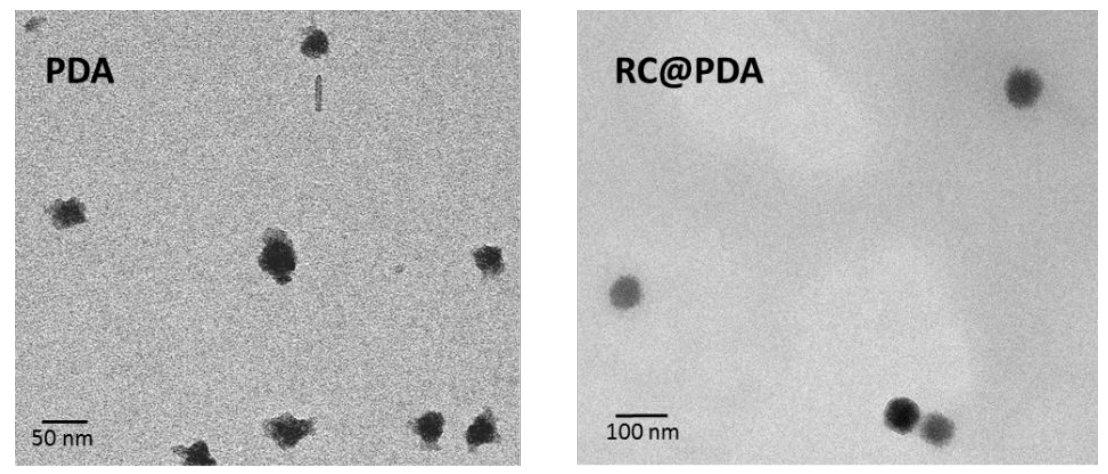
PDA nanoaggregates show a diameter of about $50 \mathrm{~nm}$ with irregular contours, while RC@PDA are spherical and have a larger size. However, these images do not prove the actual encapsulation and further evidence were obtained by DA polymerization in the presence of FITC-labelled RCs.
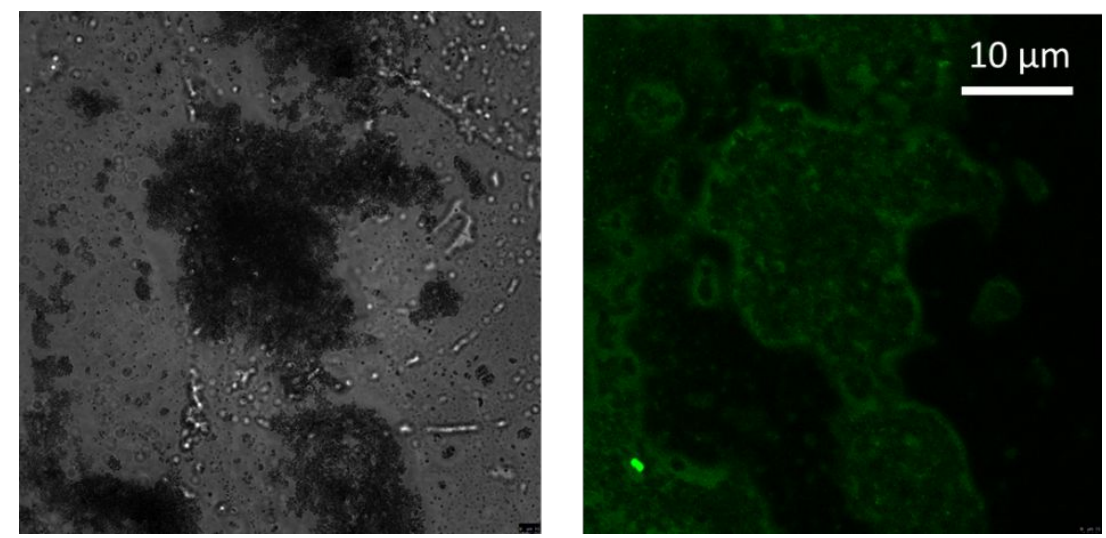

Figure 3. Confocal images of RC@PDA nanoaggregates in bright field (left) and fluorescence (right). DA $20 \mathrm{mM}$ and RCFITC $2 \mu \mathrm{M}$. Images were obtained after centrifugation and resuspension in Tris- $\mathrm{HCl} 10 \mathrm{mM}$ buffer at $\mathrm{pH} 8$.

The correspondence of PDA clusters with the FITC fluorescence (Figure 3 left and right panels respectively) represents a solid indication that the PDA nanoaggregates are loaded with RCs.

Having assessed the RC embedding in the PDA nanoaggregates maintaining its structural integrity, we moved on to the photochemical activity characterization of the protein.

Upon photon absorption, a charge separation reaction is promoted between the primary donor $\mathrm{D}$ and the ubiquinone acceptor complex $\mathrm{Q}$. The electron reaches the primary acceptor $\mathrm{Q}_{\mathrm{A}}$ and subsequently arrives at the secondary acceptor, $\mathrm{Q}_{\mathrm{B}}$, located in a pocket rich of polar, charged and protonable aminoacids. The differences in the binding pocket composition lead to different functional properties the two acceptors: $\mathrm{Q}_{\mathrm{A}}$ is firmly bound to the protein and acts as a one-electron acceptor while $\mathrm{Q}_{\mathrm{B}}$ is only loosely bound to its pocket and is prone to double reduction and double protonation to form a quinol that leaves the protein [27]. In isolated RCs, when the $\mathrm{Q}_{\text {в }}$ pocket is empty or inhibited by herbicides, a short saturating light pulse leads to the sudden formation of the charge separated state $\mathrm{D}^{+} \mathrm{Q}_{\mathrm{A}}^{-}$that decays in the dark through a charge recombination reaction (CRR) with a monoexponential kinetics having time constants $k_{\mathrm{AD}} \sim 10 \mathrm{~s}^{-1}$. When the $\mathrm{Q}_{\mathrm{B}}$ site becomes populated, a slow phase in the CRR appears since the back-reaction occurs mainly from the $\mathrm{D}^{+} \mathrm{Q}_{\mathrm{A}}{ }^{-}$state; indeed the direct route from $\mathrm{D}^{+} \mathrm{Q}_{\mathrm{A}} \mathrm{Q}_{\mathrm{B}}{ }^{-}$accounts for less than $5 \%$ [28] and the electron transfer to $\mathrm{Q}_{\mathrm{B}}$ diminishes the time averaged population of $\mathrm{Q}_{\mathrm{A}}{ }^{-}$. At saturating quinone concentrations, the decay is hence still monoexponential with a time constant $\tau_{\infty}$ $=\left(1+\mathrm{L}_{\mathrm{AB}}\right) / \mathrm{k}_{\mathrm{AD}} \cdot \mathrm{L}_{\mathrm{AB}}$ represents the interquinone electron transfer equilibrium constant and depends on the temperature and the solubilizing environment[29]. The larger its value, the larger is the stabilization of the $\mathrm{Q}_{\mathrm{B}}{ }^{-}$state with respect to $\mathrm{Q}_{\mathrm{A}}{ }^{-}$. For sub-saturating ubiquinone concentration, the CRR becomes biphasic:

$$
\chi\left(D^{+}\right)=A_{F} \exp \left(-k_{\mathrm{AD}} \mathrm{t}\right)+\mathrm{A}_{\mathrm{S}} \exp \left(-k_{\mathrm{S}} \mathrm{t}\right)(\text { Equation } 1)
$$

where $\chi\left(\mathrm{D}^{+}\right)$is the fraction of all species in a charge separated state. The first exponential accounts for the direct charge recombination (fast decay with amplitude $A_{F}$ and time 
constant $k_{\mathrm{AD}}$ ), the second exponential (slow decay with amplitude $\mathrm{A}_{\mathrm{S}}$ ) accounts for the indirect charge recombination with a time constant $k_{\mathrm{S}}$ that may depend on the population of $\mathrm{Q}_{B}-$ site. $\mathrm{A}_{\mathrm{S}}$ is roughly proportional to the fraction of $\mathrm{Q}_{B}$-populated RCs. The CRR can be easily followed spectrophotometrically at $865 \mathrm{~nm}$, where the dimer band is bleached upon $\mathrm{D}^{+}$formation and recovers as the reaction proceeds. The CRR traces of RC@PDA suspension corresponding to the spectra in Figure 1 are shown in Figure 4. The CRR trace in the original TLE buffer is also shown for comparison.

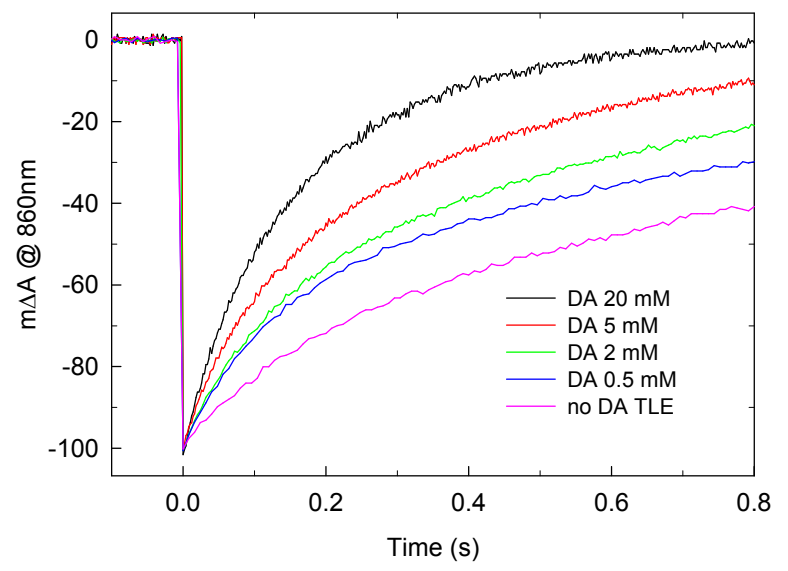

Figure 4. Charge recombination reaction traces detected at $865 \mathrm{~nm}$ in RC@PDA suspensions after overnight incubation with different DA concentrations as detailed in the legend.

As DA concentration increases, the CRR decays accelerate. All CRR traces have been fitted to Equation 1 fixing $k_{A D}$ to $8 \mathrm{~s}^{-1}$ and adding a constant term $\mathrm{c}$ to account for small signal drifts (always $\leq 3 \%$ of the overall signal). In Table 1 , the best fit parameters are given.

Table 1. Best fit parameters to Equation 1 of Traces in figure 4

\begin{tabular}{|l|l|l|l|l|l|}
\hline Parameter & TLE & DA 0.5 mM & DA 2 mM & DA 5 mM & DA 20 mM \\
\hline $\mathrm{A}_{\mathrm{S}}$ & $0.76 \pm 0.07$ & $0.58 \pm 0.06$ & $0.59 \pm 0.06$ & $0.60 \pm 0.06$ & $0.31 \pm 0.05$ \\
\hline$k_{S}\left(\mathrm{~s}^{-1}\right)$ & $0.87 \pm 0.01$ & $0.92 \pm 0.01$ & $1.32 \pm 0.02$ & $2.03 \pm 0.04$ & $2.5 \pm 0.5$ \\
\hline
\end{tabular}

The CRR acceleration is generally due to a diminished $\mathrm{Q}_{\mathrm{B}}$ activity as discussed above. The amplitude of the slow phase $\mathrm{A}_{\mathrm{S}}$ decrease, passing from $76 \%$ in bare $\mathrm{RC}$ down to $31 \%$ in RC@PDA prepared from DA $20 \mathrm{mM}$. The rate $k_{S}$, increases from $0.87 \mathrm{~s}^{-1}$ in bare RCs to $2.5 \mathrm{~s}^{-1}$ for DA $20 \mathrm{mM}$.

The decrease of $A_{S}$ is indicative of a progressive emptying of the $Q_{B}$ site, possibly because of the quinone dissolution in the surrounding PDA matrix. The $k_{S}$ increase is a consequence of the decrease of the $\mathrm{L}_{\mathrm{AB}}$ value, indicating that the semiquinone $\mathrm{Q}_{\mathrm{B}}{ }^{-}$is destabilized with respect to $\mathrm{Q}_{\mathrm{A}}^{-}$. Altogether, these results indicate that excessive DA concentration is detrimental for secondary acceptor functionality, so that optimal RC/DA ratio must be chosen for effective incorporation without harming the protein photoactivity.

The final step of this work was to asses the ability of RC@PDA to perform the full photocycle of the RC and eventually produce photocurrents. The in vivo photocycle involves the reduction of the $\mathrm{UQ}_{10}$ sitting in the $\mathrm{Q}_{\mathrm{B}}$ pocket to ubiquinol in a two-step 
reaction with the simultaneous oxidation of two cyt $\mathrm{c}^{2+}$ proteins. The formation of ubiquinol requires the uptake from the solution of two $\mathrm{H}^{+}$ions. Photocurrent can be generated in RC-based photoelectrochemical cells (PECs) by photo-activating the RC photocycle in the presence of suitable electron donors and acceptors that can be either the natural ones or other molecules with the appropriate redox potential and chemical reactivity. We used FenMeOH as metalloorganic electron donor in place of cytochrome and decylubiquinone as electron acceptor in place of $\mathrm{UQ}_{10}$.

In the classical three-electrode configuration, no current flows in dark conditions at the open circuit voltage (OCV). Upon illumination a photocurrent appears as the result of the equilibrium displacement arising from the donor oxidation and the acceptor reduction, and their subsequent reaction at the electrodes. The photocurrent will be either cathodic or anodic depending upon the reaction at the WE; cathodic photocurrent are recorded due to the reduction of the oxidised donor, while anodic photocurrent are recorded due oxidation of the reduced electron acceptor.

The RC@PDA used in these experiments were prepared in slightly different conditions with respect to the above presented experiments. A solution of RC $1 \mu \mathrm{M}$ was incubated for $1 \mathrm{~h}$ with $5 \mathrm{mM}$ DA in Tris $250 \mathrm{mM} \mathrm{pH} \mathrm{8.0.} \mathrm{The} \mathrm{higher} \mathrm{buffer} \mathrm{concentration} \mathrm{resulted}$ in the production of more PDA nanoaggregates with respect to those produced using Tris $10 \mathrm{mM}$, as inferred from the increased scattering in the optical spectrum at the end of incubation time (not shown). Upon ultracentrifugation, the spectroscopical analysis revealed that half of the RC remains in the supernatant (hence not entrapped) and half is found in the resuspended pellet that contains only entrapped RC. These RC@PDA nanoparticles were diluted 1:8 in the electrochemical cell described in the experimental section, obtaining a final RC concentration of $50 \mathrm{nM}$. The obtained photocurrent is shown in Figure 5.

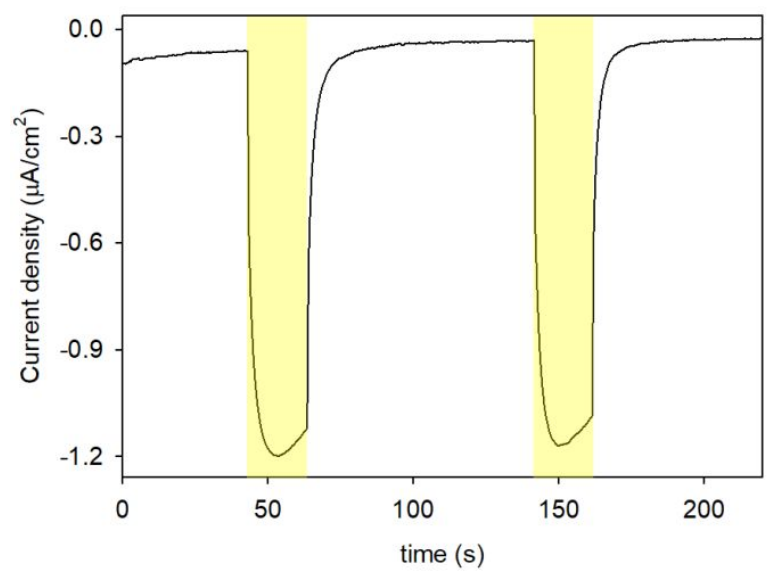

Figure 5. Photocurrent detected using RC@PDA pellet resuspended in $\mathrm{P}_{100} \mathrm{TX}_{0.03}$ with $[\mathrm{RC}]=50 \mathrm{nM},[\mathrm{FcnMeOH}]=$ $300 \mu \mathrm{M},[\mathrm{dQ}]=100 \mu \mathrm{M}$ and applied bias $-0.1 \mathrm{~V}$. The illumination period is highlighted in yellow.

The negative sign of the photocurrent indicates that a cathodic process is occurring at the WE, $i$. e. the reduction of the photooxidized species $\mathrm{FcnMeOH}^{+}$. The signal intensity of about $1.2 \mu \mathrm{A} / \mathrm{cm}^{2}$ is comparable with that obtained with the same concentration of $\mathrm{RC}$ dissolved in Triton X-100 micelles, indicating that the RC confinement within the PDA nanoaggregates does not impairs its ability to interact with the diffusive mediators. 


\section{CONCLUSION}

Nanoaggregates formed by reaction center encapsulated in polydomanine were prepared in different experimental conditions exploiting the spontaneous polymerization of domanine in slightly alkaline conditions and in the presence of dissolved oxygen. The protein preserves its structural integrity, as well as its photoactivity in these nanoaggregates.

Transient absorption spectroscopy kinetics show the progressive extraction of final electron acceptor $\mathrm{UQ}_{10}$ from the $\mathrm{Q}_{\mathrm{B}}$ binding site and a destabilization of the semiquinone $\mathrm{Q}_{\mathrm{B}}{ }^{-}$with respect to $\mathrm{Q}_{\mathrm{A}}{ }^{-}$, suggesting that the starting $\mathrm{RC} / \mathrm{DA}$ ratio require a fine tuning that depends on the application goal.

Photocurrents obtained with RC@PDA hybrid systems are comparable with those obtained with bare RCs, showing that encapsulation does not impair redox reactions between the protein and the electrochemical mediators

\section{References:}

[1] F. Milano, A. Punzi, R. Ragni, M. Trotta, G. M. Farinola, Adv. Funct. Mater. 2019, 29, 1805521.

[2] S. Bajracharya, M. Sharma, G. Mohanakrishna, X. Dominguez Benneton, D. P. B. T. B. Strik, P. M. Sarma, D. Pant, Renewable Energy 2016, 98, 153-170.

[3] D. N. Roxby, Z. Yuan, S. Krishnamoorthy, P. Wu, W.-C. Tu, G.-E. Chang, R. Lau, Y.-C. Chen, Advanced Science 2020, 1903707.

[4] A. Kumar, G. D. Park, S. K. S. Patel, S. Kondaveeti, S. Otari, M. Z. Anwar, V. C. Kalia, Y. Singh, S. C. Kim, B.-K. Cho, J.-H. Sohn, D. R. Kim, Y. C. Kang, J.-K. Lee, Chem. Eng. J. 2019, 359, 1252-1264.

[5] J. Gao, W. Kong, L. Zhou, Y. He, L. Ma, Y. Wang, L. Yin, Y. Jiang, Chem. Eng. J. 2017, 309, 70-79.

[6] Y. Du, J. Gao, L. Zhou, L. Ma, Y. He, X. Zheng, Z. Huang, Y. Jiang, Adv. Sci. 2019, 6, 1801684.

[7] S. Cho, S.-H. Kim, J. Colloid Interface Sci. 2015, 458, 87-93.

[8] J. Liebscher, R. Mrowczynski, H. A. Scheidt, C. Filip, N. D. Hadade, R. Turcu, A. Bende, S. Beck, Langmuir 2013, 29, 10539-10548.

[9] Q. Lyu, N. Hsueh, C. L. L. Chai, Langmuir 2019, 35, 5191-5201.

[10] M. Lo Presti, M. M. Giangregorio, R. Ragni, L. Giotta, M. R. Guascito, R. Comparelli, E. Fanizza, R. R. Tangorra, A. Agostiano, M. Losurdo, G. M. Farinola, F. Milano, M. Trotta, Adv. Electron. Mater. 2020, 2000140.

[11] Q. Ye, F. Zhou, W. Liu, Chem. Soc. Rev. 2011, 40, 4244-4258.

[12] N. F. Della Vecchia, A. Luchini, A. Napolitano, G. D'Errico, G. Vitiello, N. Szekely, M. d'Ischia, L. Paduano, Langmuir 2014, 30, 9811-9818.

[13] M. Trotta, F. Milano, L. Nagy, A. Agostiano, Materials Science \& Engineering. C, Biomimetic and Supramolecular Systems 2002, 22, 263-267.

[14] E. Musazade, R. Voloshin, N. Brady, J. Mondal, S. Atashova, S. K. Zharmukhamedov, I. Huseynova, S. Ramakrishna, M. M. Najafpour, J.-R. Shen, B. D. Bruce, S. I. Allakhverdiev, Journal of Photochemistry and Photobiology C: Photochemistry Reviews 2018, 35, 134-156.

[15] E. Altamura, R. Fiorentino, F. Milano, M. Trotta, G. Palazzo, P. Stano, F. Mavelli, Biophys. Chem. 2017, 229, 46-56.

[16] M. Chatzipetrou, F. Milano, L. Giotta, D. Chirizzi, M. Trotta, M. Massaouti, M. R. Guascito, I. Zergioti, Electrochem. Commun. 2016, 64, 46-50.

[17] M. Di Lauro, S. la Gatta, C. A. Bortolotti, V. Beni, V. Parkula, S. Drakopoulou, M. Giordani, M. Berto, F. Milano, T. Cramer, M. Murgia, A. Agostiano, G. M. Farinola, M. Trotta, F. Biscarini, Adv. Electron. Mater. 2020, 6, 1900888.

[18] W. Wang, J. Chen, C. Li, W. Tian, Nat. Commun. 2014, 5, 4647.

[19] R. R. Tangorra, A. Antonucci, F. Milano, S. la Gatta, A. Operamolla, R. Ragni, A. Agostiano, M. Trotta, G. M. Farinola, MRS Proceedings 2015, Mrsf14-1722, f05-51.

[20] R. A. Isaacson, F. Lendzian, E. C. Abresch, W. Lubitz, G. Feher, Biophys. J. 1995, 69, 311322. 


\section{Page 9 of 9}

[21] E. Asztalos, G. Sipka, M. Kis, M. Trotta, P. Maroti, Photosynth Res 2012, 112, 129-140.

[22] V. De Leo, L. Catucci, A. E. Di Mauro, A. Agostiano, L. Giotta, M. Trotta, F. Milano, Ultrason. Sonochem. 2017, 35, 103-111.

[23] T. Cardona, Photosynth. Res. 2015, 126, 111-134.

[24] J. P. Allen, G. Feher, T. O. Yeates, H. Komiya, D. C. Rees, Proceedings of the National Academy of Sciences 1988, 85, 8487-8491.

[25] F. Milano, R. R. Tangorra, A. Agostiano, L. Giotta, V. De Leo, F. Ciriaco, M. Trotta, MRS Advances 2018, 3, 1497-1507.

[26] H. Lee, S. M. Dellatore, W. M. Miller, P. B. Messersmith, Science 2007, 318, 426-430.

[27] F. Milano, L. Gerencser, A. Agostiano, L. Nagy, M. Trotta, P. Maroti, J. Phys. Chem. B 2007, $111,4261-4270$.

[28] D. Kleinfeld, M. Y. Okamura, G. Feher, Biochim. Biophys. Acta 1984, 766, 126-140.

[29] A. Agostiano, F. Milano, M. Trotta, Eur. J. Biochem. 1999, 262, 358-364. 